\title{
An andrologist in the front line Covid-19 team
}

\author{
Emanuele Baldassarre ${ }^{1}$
}

Received: 3 May 2020 / Revised: 6 May 2020 / Accepted: 19 May 2020 / Published online: 4 June 2020

(c) The Author(s), under exclusive licence to Springer Nature Limited 2020

\section{To the Editor:}

I read with interest the recently published article by Rizzo et al. [1] and I would like to share my experience as an Italian andrologist working at the front line in Covid-19 wards in Valle d'Aosta, the Italian region with the highest rate of infection with coronavirus based on the number of residents.

Since the middle of March 2020 our Hospital, (Umberto Parini Hospital, Aosta, Italy) has begun a gradual transformation into a "Covid-19 Unit" with the medical and surgical wards turned into COVID-19 wards at different care intensities.

Our Department of Surgery has been transformed as well. In particular, the Urology/Andrology ward, that I lead, underwent a deep transformation: medical rooms, warehouses, the kitchen, the aisles have been transformed into rooms to allow the medical staff to adopt the safe procedures before and after visiting patients, single and double rooms to treat and isolate positive COVID-19 patients, areas for storage and disposal of specific COVID-19 equipment. At the same time the working life of physicians and nurses has been highly affected by the new policies and procedures of the hospital and the pandemic itself.

Being a clinician, expert in elective surgery, I do believe that it has been extremely important to try to manage as soon as possible the stress. I took advantage of training courses in the hospital aiming at learning about mechanical ventilation, online trainings courses available on the website and, personally, I was lucky to have as my own teacher my wife, a specialist in Emergency Medicine.

After a few days from the starting of emergency in our region, our Head of Department looked for volunteers from

Emanuele Baldassarre

ebaldas75@gmail.com

1 Department of Urology, Umberto Parini Hospital, Aosta (I), Italy the different Surgical Departments to be assigned to various Covid wards.

I was assigned to the Geriatric unit where "suspected" Covid-19 patients were hospitalized before being transferred into a medium-intensive Covid-19 care unit, if later confirmed as "positive cases".

I learnt as essential part of this experience how we all can adapt to the most unexpected situation. You learn to work for $8 \mathrm{~h}$ shifts wearing personal protective equipment to know a new job in a short time, to interact with health care workers you barely know or with colleagues who were recruited at the last time, without a specific training or coming from the most different departments. Indeed, you have to work with internists, oncologists, geriatricians, gastroenterologists, clinicians who have a completely different approach in managing the patient compared to yours, and even with a jargon that is not too familiar to you.

How can an andrologist work in a Covid Area? How can I make a real contribution in supporting and treating patients? There is certainly no rule for this and I believe a great help comes from the previous experience and the spirit of adaptability of everyone.

However, I would like to point out some aspects that have helped me during this hard time and that may help those who find themselves in my situation:

- Do not waste time: learn the basics of ventilation, the use of ventilation systems and their location within the department.

- Ask colleagues from the Covid task force to disseminate procedures and videos on the correct use of personal protective equipment within the group of doctors involved, constantly sending feedback and updates.

- During your free time, necessary to maintain your mental and physical health (family routine, sports, hobbies...) attend webcalls of the various groups (anesthesiologists, infectious disease specialists, epidemiologists, etc) to keep upwith a new status of mind and language that you will need in the Covid-19 ward and with the news colleagues. 
- Equip the Covid-19 ward with visual material, quickly accessible by everyone, such as creating a folder on Google Drive with the relative documentation or add the colors of the different Venturi mask devices on Desktop.

- Keep in mind that within the Covid-19 department you should not carry with your mobile devices, books, notes, and personal items (clothes, coats, shoes, rings, bracelets...). You cannot any longer carry anything personal with you as you were used to do before.

- Stick as much as possible to staff protocols, avoiding personal ideas not previously shared with the group, in order to avoid unnecessary delays and changes in the therapy. Herein, any clinician/surgeon is no longer the center of attention, but is part of a team in which the infectious and pneumological problems are of primary importance.

- Be ready to work with 2-3 pairs of gloves with reduced sensitivity during invasive maneuvers, such as performing arterial sampling or positioning of devices. It can be a good training to take venous accesses together with the nurses a few days before being an active member of a Covid-19 unit.

- When working in a Covid-19 unit, as surgeon, you can use your own skills at different levels: from the management of simplest problems, such as a tracheostomy, until the management of complex medications such as thoracentesis or paracentesis or dealing with procedures in patients with vascular problems.

- Always be available to talk to relatives on the phone being aware of their loneliness and isolation in which their loved ones are. Be ready to have enough time to talk to them and do not consider it as a "waste time".
- Do not lose control with your colleagues, who are probably more tired than you or perceive fear differently. Being open to listen to whatever advice or suggestions your colleagues may have: we need to get out of the mentality of a defensive medicine.

- Be curious and not be ashamed to ask even younger medical colleagues for advice. Covid-19 is " a democratic disease": we are all equal behind that FFP2/FFP3 masks and whoever knows how to improve the way of working in a team with a spirit of adaptability wins.

Beside these tips, this period it was not easy to pass and the initiatives implemented by the Italian Andrology Society were important because they allowed me to maintain mental with the world of Andrology and reduced the risk of burnout.

\section{Compliance with ethical standards}

Conflict of interest The author declares that they have no conflict of interest.

Publisher's note Springer Nature remains neutral with regard to jurisdictional claims in published maps and institutional affiliations.

\section{References}

1. Rizzo M, Liguori G, Verzé P, Palumbo F, Cai T, Palmieri A. How the andrological sector suffered from the dramatic Covid 19 outbreak in Italy: supportive initiatives of the Italian Association of Andrology (SIA). Int J Impot Res. 2020;1-2. [published online ahead of print, 2020 Apr 23]. 